Meta

Journal des traducteurs

Translators' Journal

\title{
Figement et intensité en grec moderne
}

\section{Zoé Gavriilidou}

Volume 53, numéro 2, juin 2008

La traduction des séquences figées

The Translation of Frozen Sequences

URI : https://id.erudit.org/iderudit/018524ar

DOI : https://doi.org/10.7202/018524ar

Aller au sommaire du numéro

Éditeur(s)

Les Presses de l'Université de Montréal

ISSN

0026-0452 (imprimé)

1492-1421 (numérique)

Découvrir la revue

Citer cet article

Gavriilidou, Z. (2008). Figement et intensité en grec moderne. Meta, 53(2),

365-377. https://doi.org/10.7202/018524ar

\section{Résumé de l'article}

Dans cet article, nous nous proposons d'étudier l'intensité repérée dans des constructions figées en grec moderne, en dégageant les caractéristiques de l'intensité. Nous mettons l'accent sur l'intensité dans a) les déterminants figés

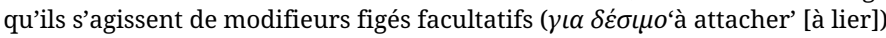

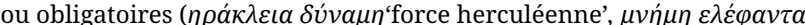

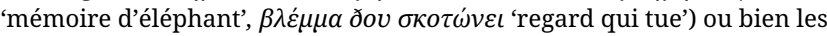
prédéterminants composés figés ( $\omega \kappa \varepsilon \alpha v o ́ \varsigma \delta \alpha \kappa \rho u ́ \omega v^{\prime}$ océan de larmes') ; b) les

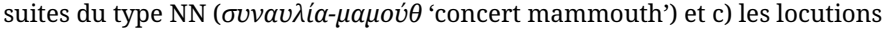

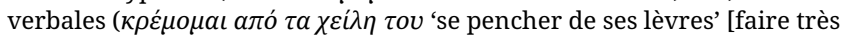
attention]). Afin de résoudre quelques problèmes de traduction liés à l'intensité, nous employons la notion théorique de représentation métalinguistique (Buvet, à paraître b), pour proposer des traductions correctes pour les expressions figées qui désignent l'intensité.
Ce document est protégé par la loi sur le droit d'auteur. L’utilisation des services d’Érudit (y compris la reproduction) est assujettie à sa politique d'utilisation que vous pouvez consulter en ligne.

https://apropos.erudit.org/fr/usagers/politique-dutilisation/ 


\title{
Figement et intensité en grec moderne
}

\author{
ZOÉ GAVRIILIDOU \\ Democritus University of Thrace, Komotini, Greece \\ zoegab@otenet.gr
}

\section{RÉSUMÉ}

Dans cet article, nous nous proposons d'étudier l'intensité repérée dans des constructions figées en grec moderne, en dégageant les caractéristiques de l'intensité. Nous mettons l'accent sur l'intensité dans a) les déterminants figés qu'ils s'agissent de modifieurs figés facultatifs (

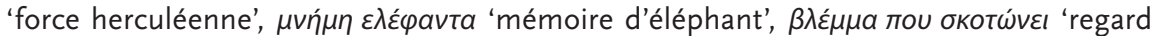
qui tue') ou bien les prédéterminants composés figés ( $\omega$ каavó $\delta a \kappa p u ́ \omega v$ 'océan de lar-

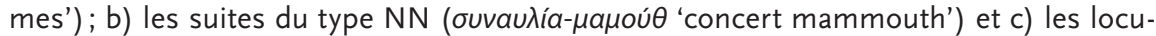

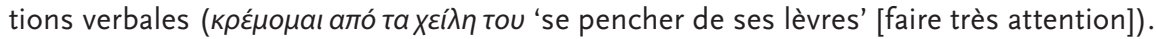
Afin de résoudre quelques problèmes de traduction liés à l'intensité, nous employons la notion théorique de représentation métalinguistique (Buvet, à paraître b), pour proposer des traductions correctes pour les expressions figées qui désignent l'intensité.

\section{ABSTRACT}

In this paper we deal with intensity found in frozen expressions of modern Greek. We take into account three different kinds of frozen expressions: a) Frozen determiners, b) Noun-Noun constructions, c) Verbal constructions. We put forward the idea that Noun-Noun constructions have similar semantic and syntactic characteristics as frozen determiners when marking intensity, while verbal constructions are of a completely different nature. We also mention some translation problems caused by frozenness and intensity and we use metalinguistic representations of predicates, in order to achieve correct translations of frozen expressions marking intensity.

\section{MOTS-CLÉS/KEYWORDS}

intensité, figement, représentation métalinguistique, quantifieur, gradation

\section{Introduction}

L'intensité est un des phénomènes les plus courants dans les langues. Elle peut être exprimée à l'aide de plusieurs procédés linguistiques. Les exemples de cas d'intensité abondent aussi dans la partie figée de la langue.

Nous avons examiné ailleurs (Gavriilidou 2004) les verbes supports intensifs en grec moderne. Le présent travail veut contribuer à l'élucidation du concept d'intensité repéré dans des constructions figées en grec moderne. Dans ce but, nous essayons, dans un premier temps, de dégager les caractéristiques de l'intensité. Ensuite, nous mettons l'accent sur l'intensité dans les séquences figées. Toutefois, il est impossible d'étudier de façon exhaustive toutes les sortes de figement. C'est pourquoi, nous étudions de près l'intensité dans: 1 . les déterminants figés (modifieurs figés facultatifs

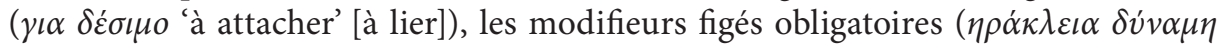

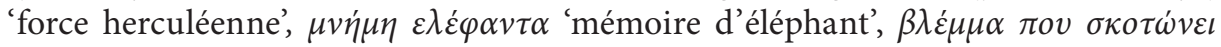
'regard qui tue'), les prédéterminants composés figés ( $\omega \kappa \varepsilon \alpha v o ́ \varsigma \delta \alpha \kappa \rho v i \omega v$ 'océan de larmes'); 2 . les suites du type NN ( $\sigma v v \alpha v \lambda i \alpha-\mu \alpha \mu o v \theta \theta$ 'concert-mammouth'); et 3. les 


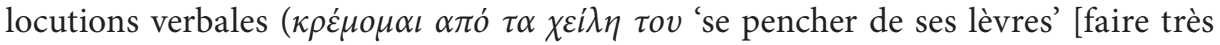
attention]). Enfin, nous insistons sur quelques problèmes de traduction liés à l'intensité et nous employons la notion théorique de représentation métalinguistique (Buvet à paraître b) afin de proposer des traductions correctes pour les expressions figées qui désignent l'intensité.

\section{L'intensité}

\subsection{Les caractéristiques de l'intensité}

Il existe dans les langues des expressions qui renforcent le sens d'un mot, autrement

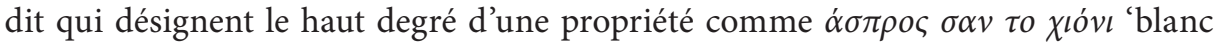

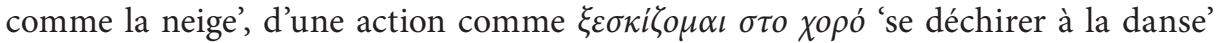
[danser beaucoup] ou d'un état comme $\pi \lambda \eta \mu \mu v \rho i \zeta \omega \alpha \pi o ́ ~ \chi \alpha \rho \alpha ́$ 'être inondé de joie'. Ces expressions sont des expressions intensives ${ }^{1}$. Dans cette étude, nous appelons intensificateur un type particulier de modifieur dont la fonction est d'introduire une

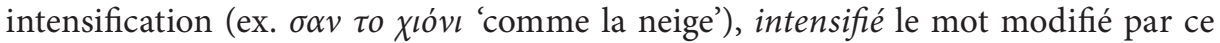
modifieur (ex. $\alpha \sigma \pi \rho \circ \varsigma$ 'blanc') et expression intensive la structure intensificateur-

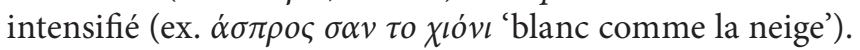

Il est impossible de parler d'intensité, sans évoquer la notion de quantité, les notions d'intensification et de quantification n'étant pas toujours clairement dissociables. Pour Romero (2001: 11), «la notion de quantité abstraitement conçue peut s'appliquer soit à la substance (c'est le sens concret courant de quantité), soit à la qualité (on parle alors de degré ou d'intensité)». Dans le présent travail, nous étudions soit des quantifieurs ( $\omega \kappa \varepsilon \alpha v o ́ \varsigma \delta \alpha \kappa \rho v ́ \omega v$ 'un océan de larmes'), soit des intensifs

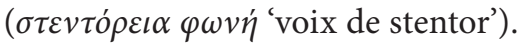

Malgré le nombre élevé d'auteurs qui ont essayé de proposer des définitions pour la notion d'intensité, il n'y a pas à notre connaissance de définition unanime, dans la mesure où l'intensité peut être définie en se basant chaque fois sur des critères divers. C'est pour cette raison que nous préférons discuter certaines de ses caractéristiques, au lieu d'essayer de fournir une définition de cette notion.

- L'intensité exprime une affection:

Selon Szende (1999: 63), «l'acte d'évaluation peut avoir un contenu affectif avec une nuance laudative ou péjorative; l'intensité est souvent obtenue en renforçant le positif ou le négatif». En parlant en termes d'analyse du discours, les expressions intensives sont évaluatives (pour l'évaluation, cf. Hunston et Thompson 2000) et les phrases dans lesquelles elles figurent ont pour but d'exprimer l'opinion de l'auteur et par conséquent de refléter son système de valeurs, bâtir et maintenir des relations entre l'auteur et le lecteur (par exemple persuader, convaincre le lecteur), et enfin organiser le discours.

- L'intensité n'affecte pas tous les mots:

L'intensité affecte plusieurs types morphologiques (noms, verbes, adjectifs) ou sémantiques (actions, états, événements) de prédicats, mais il en existe plusieurs qui ne sont pas intensifiables. La question qu'il faut se poser alors est que signifie «être intensifiable». Pour un grand nombre d'auteurs, le concept d'intensificabilité va de pair avec 
celui de gradation (Cruse 1986) ou d'échelle (Romero 2001). Et quand on parle de gradation, c'est l'adjectif qualificatif qui vient en premier, comme étant le prototype des éléments linguistiques gradables. Il existe toutefois des noms - prédicatifs ou non - ou des verbes qui peuvent être intensifiés, malgré le fait qu'ils ne sont pas traditionnellement considérés comme gradables. Comment peut-on alors expliquer ce phénomène?

Le modèle des classes d'objets (Gross 1992), en combinaison avec la théorie du prototype, pourrait donner une explication suffisante. Ce modèle classe les prédicats dans des classes sémantiquement et syntaxiquement homogènes à l'aide de critères syntaxiques. Chaque classe ou sous-classe manifeste certaines caractéristiques partagées par ses membres (p. ex. tous les concrets ont une certaine taille, une couleur, etc.; un événement arrive quelque part, il a une certaine durée, etc.). C'est chaque fois une seule caractéristique du mot modifié qui est intensifiée (par exemple, dans $\tau \alpha ı v i \alpha-\pi o \tau \alpha \mu o ́ \varsigma$ 'film-fleuve', c'est le paramètre de la durée du film qui est intensifié). Quant au nom intensifiant, il est souvent le prototype d'une propriété, autrement dit le meilleur exemplaire d'une classe (par exemple, dans $\eta \rho \alpha ́ \kappa \lambda \varepsilon \iota \alpha \delta$ v́v $\alpha \mu \eta$ 'force herculéenne' l'adjectif $\eta \rho \alpha ́ \alpha \lambda \varepsilon \iota \alpha$ renvoie à Hercule qui était considéré comme le prototype de l'homme fort). La notion de prototype évoquée ici permet à un mot non gradable de devenir gradable et d'accepter une intensification.

Pour éviter d'expliquer la possibilité d'intensification de noms non gradables, Cesare (2002: 16), de son côté, soutient qu'«est intensifiable tout prédicat modifiable par un intensificateur ${ }^{2} »$.

- L'intensité va souvent de pair avec l'atténuation:

Selon Cruse (1986), la plupart des 'échelles' (p.ex. la taille, la hauteur, la vitesse) forment un continuum dont les deux extrêmes, le point positif et le point négatif, sont occupés par des termes intensifs lexicalisés, qui sont entre eux des antonymes comme

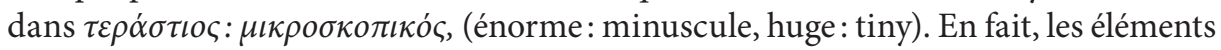
linguistiques employés pour marquer le pôle négatif d'une échelle ajoutent une information d'un degré faible, et sont appelés des atténuateurs. Selon Szende (1999: 63), "là où un adjectif fonctionne comme opérateur d'intensification, un autre adjectif peut intervenir comme opérateur d'atténuation [...] L'intensificateur va souvent de pair avec un atténuateur et l'intensification va souvent de pair avec l'atténuation.» De tout ce qui est dit, on conclut que l'intensification est une variation du degré, qui comprend tant l'augmentation que la diminution.

- L'intensité déclenche une redondance sémique:

Pour Mejri (1994: 113), une redondance sémique (par ellipse, ou par sélection sémique) caractérise tous les procédés intensificatoires rencontrés. «Le deuxième terme de la comparaison représente une répétition du premier, mais d'une manière indirecte.»

- L'intensité est une fonction lexicale:

D’un point de vue lexicographique, le modèle théorique Sens-Texte (Mel'čuk 1997; Polguère 1998) a pu décrire le phénomène d'intensification créé par certaines collocations d'une manière formelle, très efficace, en s'appuyant sur la notion de fonction lexicale syntagmatique Magn $^{3}$ (cf. aussi Mel'čuk 1988), qui est une fonction au sens 
mathématique, qui peut être représentée par la formule suivante: $\boldsymbol{f}(x)=y$, où $x$ est l'argument de la fonction et $y$ sa valeur. Par exemple, dans froid de canard, $\boldsymbol{f}$ est l'intensification, $x=$ froid, et $y=$ de canard. Cette fonction intensifie une composante sémantique particulière du mot sur lequel elle porte, c'est-à-dire qu'elle réfère à un trait sémantique particulier dans une situation donnée: ainsi, dans chute brutale, l'intensification porte sur la vitesse du déroulement du phénomène, plutôt que sur sa fréquence.

\subsection{Procédés intensificatoires en grec moderne}

Chaque langue dispose d'un certain nombre de procédés par lesquels elle marque l'intensité. Le grec moderne marque l'intensité:

a) par des procédés morphologiques: préfixation comme $\pi \alpha v v$ $\psi \eta \lambda o \varsigma$ 'très haut', suffixation $\sigma \pi \alpha v i o ́ \tau \alpha \tau o \varsigma$ 'rarissime', $\sigma \pi \imath \tau \alpha \rho o ́ v \alpha$ 'maison énorme', composition $\varepsilon \tau \alpha \iota \rho i \alpha-$ $\mu \alpha \mu о v \theta$ 'entreprise-mammouth', $\gamma \iota \gamma \alpha v \tau o \alpha \varphi i \sigma \alpha$ 'affiche géante';

b) par des procédés syntaxiques (essentiellement par détermination): adjonction d'ad-

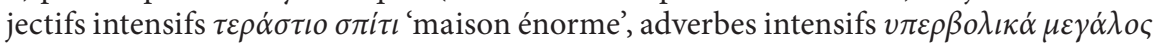

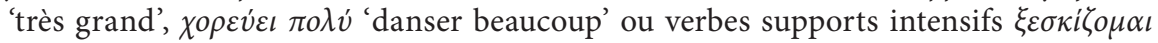

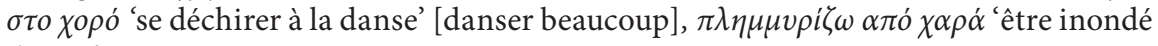
de joie';

c) par des procédés prosodiques (utilisation de certaines intonations ou accents emphatiques, répétition de mots, etc.);

d) par des collocations $\chi v ́ v \omega \mu \alpha v ́ \rho o \delta \alpha ́ \alpha \rho v$ 'verser larme noire' [pleurer à larmes chaudes].

On retrouve plus ou moins les mêmes mécanismes en français cf. boire comme un Polonais, fièvre de cheval, ultra-confidentiel, rarissime, etc., ou dans d'autres langues comme l'espagnol: saltar de allegria 'sauter de joie', sueldo de hambre 'salaire de misère', paciencia de santo 'patience d'ange', l'anglais: top secret, super-intelligent, etc. Toutefois, même si les mécanismes sont plus ou moins les mêmes, les unités lexicales qui entrent dans les structures équivalentes, afin de créer des expressions intensives ne sont pas les mêmes dans les différentes langues. Par exemple en grec, on emploie

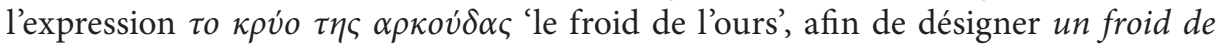
canard. Ce phénomène pose des problèmes de traduction, quand il s'agit de traduire les expressions intensives d'une langue à l'autre.

Après avoir énuméré certaines des caractéristiques de l'intensité, avoir vu les procédés principaux d'intensification en grec moderne et avoir suggéré quelques problèmes de traduction des expressions intensives d'une langue à l'autre, nous nous intéressons dans ce qui suit au comportement des éléments intensifiants et intensifiés dans le cadre des expressions figées intensives, afin de pouvoir proposer une solution aux problèmes de traduction mentionnés ci-dessus.

\section{Figement et intensité}

Il existe dans les langues un grand nombre de formules appelées expressions figées dont les principales caractéristiques sont les suivantes (Gross 1996; Mejri 1996) : a) la polylexicalité; b) l'opacité sémantique; c) le blocage de leurs propriétés transformationnelles; d) la non-actualisation de leurs éléments; e) le blocage des paradigmes 
synonymiques; f) la non-insertion d'éléments, etc. Bien sûr, le figement est un phénomène scalaire (Gross 1988), ce qui veut dire qu'il y a parfois une certaine liberté lexicale, une possibilité de paradigme ou de transformation. Le figement absolu est un cas marginal.

Un examen minutieux des expressions figées nous permet d'observer des cas très hétérogènes du point de vue de la forme qui pourraient, cependant, constituer une même catégorie, dans la mesure où toutes expriment l'idée du haut degré de l'intensité. On dirait que le figement est un procédé prototypique de la langue pour marquer l'intensité.

Parmi tous les cas d'expressions figées intensives, nous étudierons plus particulièrement les déterminants figés intensifs, les suites du type NN et les locutions verbales, afin de relever les différences ou convergences sémantico-syntaxiques éventuelles de ces trois types d'expression de l'intensité.

\subsection{Les déterminants figés}

La détermination d'un substantif tête est constituée d'un prédéterminant et d'un éventuel modifieur. Sont considérés comme prédéterminants les éléments qui précèdent la tête du groupe nominal, tandis que tous les autres éléments sont des modifieurs (Buvet, à paraître).

Selon G. Gross (1996), la détermination peut être simple ou complexe. La détermination complexe est subdivisée en association d'éléments déterminatifs et de détermination composée. L'association d'éléments déterminatifs peut être libre (e.g.

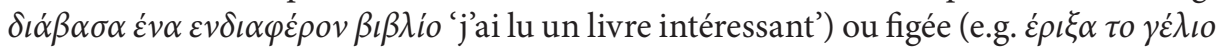
$\tau \eta \varsigma \alpha \rho \kappa o v ́ \delta \alpha \varsigma$ ‘j’ai jeté le rire de l'ours' [j’ai ri énormément]). Dans le premier cas, l'association des éléments déterminatifs suit les restrictions de sélection imposées dans les phrases (choix d'un déterminant spécifique selon le type de prédicat, ordre des mots, etc.), tandis que dans le second, le figement est produit par une association sui generis entre un prédéterminant, un modifieur et un substantif donné (cf. la relation entre l'article défini neutre $\tau o$, le groupe nominal au génitif $\tau \eta \varsigma \alpha \rho \kappa o v ́ \delta \alpha \varsigma$ 'de

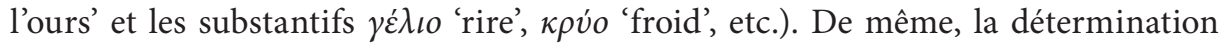

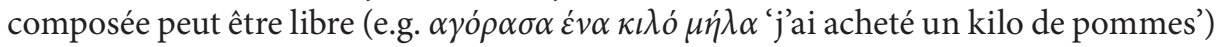
ou figée (e.g. $\chi v ́ v \omega \omega \kappa \varepsilon \alpha v o v ́ \varsigma$ $\delta \alpha \kappa \rho v ́ \omega v$ 'je verse des océans de larmes').

Ce qui nous intéresse, c'est le cas des déterminants figés, qui sont de trois types: modifieurs figés facultatifs, modifieurs figés obligatoires et prédéterminants composés figés.

\subsubsection{Les modifieurs figés facultatifs}

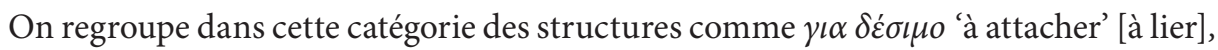

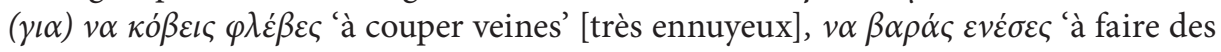

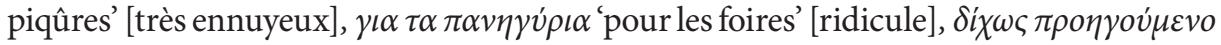

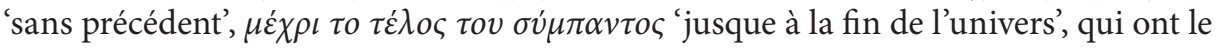

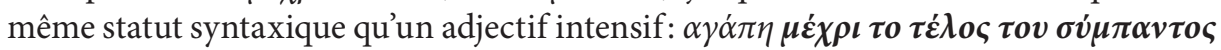

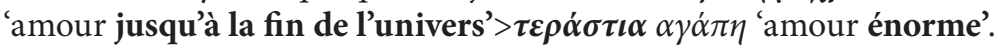

On observe souvent l'emploi de la préposition $\gamma \iota \alpha$ 'pour/à' dans les constructions de ce type. Les prépositions sont toujours suivies d'un nom, d'une phrase subjonctive ${ }^{4}$ ou d'un groupe nominal. D'un point de vue syntaxique, les groupes prépositionnels 
ainsi formés correspondent à des modifieurs qui se combinent avec divers prédéterminants pour constituer la détermination d'un substantif donné:

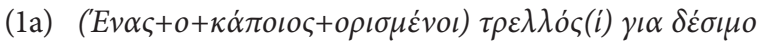
'(Un+le+quelques) fou(s) à lier'

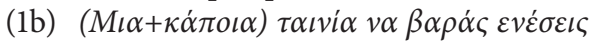
'(Un+un certain) film à faire des piqûres' [(Un+un certain) film ennuyeux]

Les groupes prépositionnels étudiés ici sont des modifieurs non liés (facultatifs):

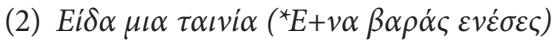

'J'ai vu un film à faire des piqûres'

[J'ai vu un film très ennuyeux]

Ils peuvent se rattacher à des prédéterminants variés comme le montrent les exemples (1a) et (1b), à la différence des modifieurs figés qui sont obligatoires et qui donnent naissance à une détermination figée, quand ils dépendent d'un seul déterminant ou type de déterminant (cf. 3.1.2) (Buvet, à paraître). Notons ici qu'il ne faut pas confondre le fait qu'un modifieur est libre ou figé avec le fait qu'il est obligatoire ou facultatif. Les modifieurs libres, tout comme les modifieurs figés, peuvent être obligatoires (cf. Luc a une peur $\left[{ }^{\star}\right.$ E+maladive]) ou facultatifs (Luc a une grippe [E+étrange]).

D'un point de vue sémantico-pragmatique, les exemples traités ci-dessus sont des expressions qui constituent une qualification intensive imagée, dans la plupart des cas arbitraire, du nom auquel ils se rattachent. Ici l'intensité ne se réfère pas à la quantité, mais à une qualité que le nom modifié porte à un degré élevé. Pour Berthelon (1955: 127), cette qualité n'est pas exprimée directement, sa valeur étant simplement suggérée, "sans nuance précise». Les modifieurs introduits par $\gamma \iota \alpha$ 'pour/à' expriment le degré de l'intensité du terme modifié en tant que conséquence de cette intensité (Romero 2001). La préposition $\gamma \iota \alpha$ 'pour/à' garde dans ces expressions son sens locatif (je suis à) d'une manière métaphorique (pour la lecture métaphorique des prépositions, cf. Lakoff 1987), ce qui fait que les exemples traités sont conçus comme exprimant l'arrivée au point extrême d'une échelle.

Dans ces exemples, il n'y a pas de redondance sémique, propriété rencontrée souvent dans d'autres intensifs, dans le sens où le terme intensificateur ne représente pas de répétition du terme intensifiant.

Les déterminants présentés dans 3.1.1 doivent être associés à d'autres éléments déterminatifs pour constituer une détermination. Examinons, dans ce qui suit, les séquences déterminatives qui forment des groupes nominaux, quand ils se combinent avec des noms.

\subsubsection{Les modifieurs figés obligatoires}

Les modifieurs obligatoires (ou liés) participant à une détermination figée sont de cinq types:

a) Les adjectifs simples

On regroupe dans cette classe des adjectifs comme $\eta \rho \alpha \dot{\kappa} \lambda \varepsilon \iota \iota \varsigma$ 'herculéen' dans

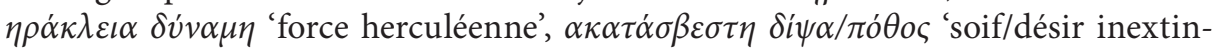

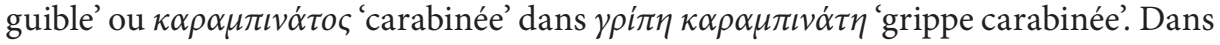


ces exemples, le critère de polylexicalité n'est pas rempli, dans la mesure où le prédéterminant et son expansion sont des formes simples. Le figement porte alors sur la relation entre le prédéterminant et son expansion (Buvet à paraître). De plus, le caractère figé dans cette catégorie résulte du fait que la séquence déterminative disjointe est une configuration stable.

La structure en question est à distinguer des noms composés du type Adj $\mathrm{N}$ ( $\delta \iota \alpha \sigma \tau \eta \mu \iota \kappa o ́ ~ \lambda \varepsilon \omega \varphi \rho \rho \varepsilon i o ~ ' n a v e t t e ~ s p a t i a l e ')$, puisque le modifieur adjectival se comporte en tant qu'adjectif qualificatif dans les exemples étudiés et en tant que pseudo-adjectif dans les noms composés (cf. Blanco, Buvet et Gavriilidou 1999).

D’un point de vue sémantique, $\eta \rho \alpha ́ \kappa \lambda \varepsilon \iota \alpha \delta v ́ v \alpha \mu \eta$ 'force herculéenne' étant une force énorme, l'intensité porte ici sur la quantité de la propriété désignée par le nom modifié. Très souvent, la mythologie grecque fournit un nombre considérable d'expressions de ce type. En fait, dans la tradition grecque, Hercule est considéré comme le prototype de la force, ou Stentor comme le prototype de l'homme à voix forte, ce qui a donné naissance dans la langue aux modifieurs $\eta \rho \alpha ́ \kappa \lambda \varepsilon \iota \alpha$ et $\sigma \tau \varepsilon v \tau o ́ \rho \varepsilon \iota \alpha$.

Selon Blanco (2002), dans certains cas, « des modifieurs figés adjectivaux pouvant être classés dans une même aire sémantique font cependant référence à des aspects différents du sémantisme du $N »$. C'est surtout le cas avec les intensifs. On trouve des exemples en grec moderne comme $\varepsilon \rho \omega \tau \alpha \varsigma$ 'amour' qui acceptent des intensifs par rap-

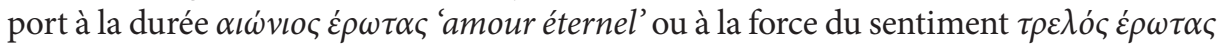
'amour fou'. Ceci arrive surtout quand le nom modifié est un prédicat non gradable. Dans ce cas, l’adjectif modifie la quantité, la durée ou la fréquence de ce prédicat.

b) Les compléments au génitif

Il s'agit de séquences comme $\mu v \eta \dot{\mu} \mu \varepsilon \lambda \hat{\varepsilon} \varphi \alpha v \tau \alpha$ 'mémoire d'éléphant' qui sont structurellement stables et qui admettent éventuellement des variations limitées à un seul de leurs constituants (cf. Buvet 1995; Blanco 1996; Gavriilidou 1998). Elles sont sémantiquement opaques et distributionnellement restreintes. Un troisième indice de leur figement est le caractère unique de la relation entre un prédéterminant et un modifieur lié. Les structures étudiées ici sont à distinguer des noms composés du type $\mathrm{N}$ de $\mathrm{N}$ ( $\zeta \omega ́ v \eta \alpha \sigma \varphi \alpha \lambda \varepsilon i ́ \alpha \varsigma$ 'ceinture de sécurité') ou des déterminants nominaux quantifieurs ( $\omega \kappa \varepsilon \alpha v o ́ \varsigma \delta \alpha \kappa \rho v ́ \omega v$ 'océan de larmes') (cf. 3.1.3) qui ont une forme de surface identique pour des raisons qu’on a mises en évidence ailleurs (Gavriilidou 1998).

Cette structure est souvent rapprochée des expressions comparatives en 'comme',

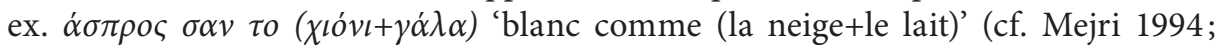
Swende 1999; Shapira 2000; Leroy 2004) où le terme introduit par comme (ou $\sigma \alpha v$ en grec moderne) "est susceptible de devenir un étalon de haut degré » (Berthelon 1955). Dans cette catégorie, le complément au génitif joue le rôle d’un complément de nom intensif qui intensifie une composante sémantique particulière du mot clé, c'est-à-dire qu'elle se réfère à un trait sémantique particulier dans une situation don-

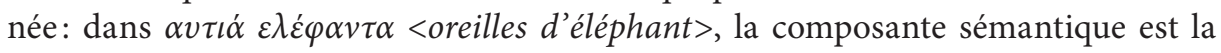
taille des oreilles, dans $\delta \iota \alpha \beta o \lambda \iota \kappa \eta$ ' $\pi \varepsilon i v \alpha<$ faim diabolique $>$ 'faim de loup', la composante sémantique intensifiée est le degré de la faim, etc. Le mot diabolique rencontré dans ce dernier exemple désigne en général quelque chose qui déplaît à un degré élevé. Toutefois, quand il entre dans des constructions de ce type, il perd sa nuance péjorative pour devenir un simple intensif. Comme nous le constatons, l'intensité dans ces exemples est quantitative. 
Très souvent, l'interprétation intensive se crée grâce à une métaphore. Une propriété ou une qualité du N1 entre en comparaison avec un N2 qui est considéré comme le prototype de cette propriété, comme P-A. Buvet et G. Gross (1995) le suggèrent en disant que «l'expression du haut degré repose sur l'association de la propriété considérée avec un terme pris comme le prototype ou le stéréotype de cette propriété». De la même façon, I. Tamba (1981 : 146) soutient que "pour donner naissance à une hyperbole, il est nécessaire que le nom ou la proposition qui sert d'étalon comparatif désigne un phénomène que ses propriétés effectives ou des conventions culturelles font tenir pour un modèle exemplaire, l'incarnation même, pourrait-on dire, de la caractéristique prise comme base de la comparaison».

Il existe toutefois des cas où les compléments de noms intensifs sont complètement arbitraires, et leur naissance est plutôt motivée par des raisons prosodiques que

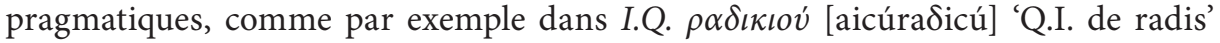
[sans Q.I. du tout].

c) Les relatives

d) Les expressions figées

e) Les subordonnées introduites par $v \alpha$

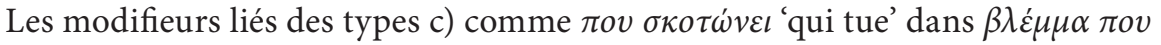
$\sigma \kappa o \tau \omega ́ v \varepsilon l$ 'regard qui tue', d) $\beta \rho \alpha ́ \sigma \varepsilon$ ó $\rho v \zeta \alpha$ dans $\kappa \alpha \tau \alpha ́ \alpha \tau \alpha \sigma \eta \beta \rho \alpha ́ \alpha \varepsilon$ ó $\rho v \zeta \alpha$ 'situation

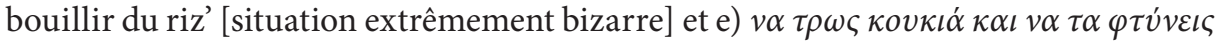
'à manger des fèves et à les cracher' [dégoûtant] sont moins fréquents que les deux premiers. Ils présentent des caractéristiques comparables à celles des adjectifs simples ou des compléments de nom au génitif et donnent également lieu à une détermination figée qui ajoute une information d'intensité.

\subsubsection{Les prédéterminants composés figés}

Il s'agit d'exemples du type N1N2génitif, N1 prép ${ }^{5}=\alpha \pi$ ó N2accusatif, N1N2accusatif,

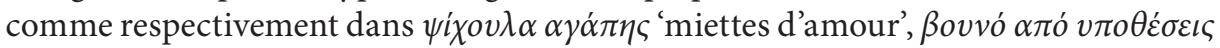

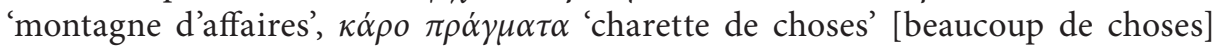
(Gavriilidou 1998). Comme nous le constatons à partir des exemples cités ci-dessus, les prédéterminants composés figés sont exclusivement des déterminants nominaux exprimant une quantité (Buvet, à paraître Blanco, Benninger 2001). Le figement qui les caractérise ressortit principalement à l'inanalysabilité de la relation entre le nom déterminatif et la tête nominale au sein du GN (Buvet à paraître). Souvent, le N1 est un nom collectif comme dans $\sigma \tau \rho \alpha \tau o ́ \varsigma \alpha \pi o ́ ~ \mu \varepsilon \rho \mu \eta ่ \gamma \kappa \iota \alpha$ 'armée de fourmis'. Selon Flaux (1999: 473), quand ils «ne sont pas tête de syntagme, c'est-à-dire lorsqu'ils fonctionnent comme des noms de quantité [...], les noms collectifs sont toujours interprétés de manière métaphorique». Une interprétation métaphorique est souvent valable pour les N1 non collectifs.

La métaphore présente dans les exemples étudiés crée une interprétation intensive. La composante sémantique intensifiée est la quantité de ce qui est désigné par

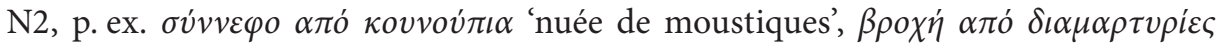
'pluie de protestations', $\kappa \dot{\mu} \mu \alpha$ ßí $\varsigma_{\varsigma}$ 'lame de violence'. Nous employons des paraphrases explicatives qui comportent un superlatif marquant le haut degré, afin de démontrer l'interprétation intensive des N1 étudiés ici: 


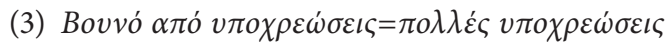

'Une montagne de responsabilités=beaucoup de responsabilités'

(4) $\Psi i \chi \chi o v \lambda \alpha \alpha \gamma \alpha \dot{\pi} \eta \varsigma=\pi o \lambda \dot{v} \lambda i \gamma \eta \alpha \gamma \alpha \dot{\pi} \eta$

'Des miettes d'amour=très peu d'amour'

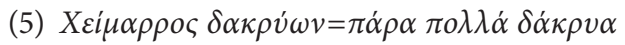

'Un torrent de larmes=beaucoup de larmes'

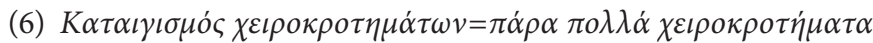

'Une rafale d'applaudissements=beaucoup d'applaudissements'

Les N1 métaphoriques peuvent désigner soit un grand nombre de/une grande quantité de N2 (ex. 3, 5, 6) (idée de renforcement) soit un petit nombre de/une petite quantité de N2 (ex. 4) (atténuation) (cf. 2). Nous avons remarqué que, quand le déterminant nominal figé désigne une grande quantité de N2, le N2 est toujours au pluriel ${ }^{6}$ (ex. 3, $5,6)$.

L'idée d'intensité devient encore plus forte quand le N1 métaphorique se met au pluriel:

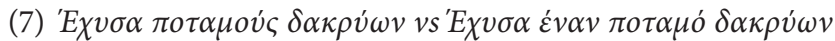

'J'ai versé des fleuves de larmes vs J'ai versé un fleuve de larmes'

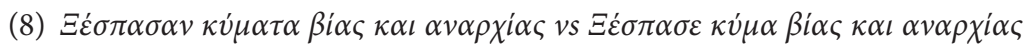

'Des lames de violence et d'anarchie ont éclaté vs Une lame de violence et d'anarchie a éclaté'

\subsection{Les suites du type NN}

Dans Gavriilidou (1997), nous avons soutenu qu'il y a, d'un point de vue syntacticosémantique, trois classes distinctes de suites Dét N1N2: les suites formées par coordination: un architecte-archéologue; les suites formées par complémentation: le centre-ville, et celles formées par attribution: un succès monstre. Une étude de la troisième catégorie sous l'angle de la théorie de grammaticalisation (Gavriilidou 2003) nous a permis de soutenir que le N2 des structures Dét N1 N2 quand il s'attache à des N1 prédicatifs, par exemple succès monstre, concert mammouth, est une sorte de modifieur figé obligatoire, et ceci, pour des critères syntaxiques et sémantiques.

De l'étude du corpus constitué, nous avons remarqué qu'un grand nombre des N2 de ce type sont des marqueurs d'intensité. Dans un exemple comme croissance champignon, la composante sémantique intensifiée est la rapidité de la croissance. En fait, le N1 croissance possède la qualité de rapidité à un degré élevé, comme le désigne l'adjonction du N2 champignon. Dans concert marathon, la composante sémantique intensifiée est la durée du concert, un concert marathon étant un concert qui dure des heures. L'idée d'intensité est aussi présente dans les cas où N2 se rattache à un

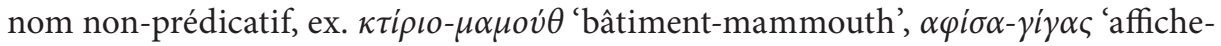
géant', $\alpha \cup \tau i-\alpha \varepsilon \rho o \pi \lambda \alpha$ vo 'oreille-avion' très grande oreille. En général, dans ce type d'exemples, l'intensité exprimée par N2 porte sur une qualité que le N1 possède à un degré élevé.

On retrouve ici un procédé métaphorique: l'expression de la ressemblance évoque un rapprochement par comparaison du N1 avec le N2, à l'aide d'une sélection sémique, comme les paraphrases suivantes nous permettent de le constater:

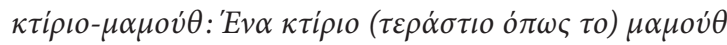

'bâtiment-mammouth: Un (bâtiment énorme comme le) mammouth' 
$\pi o ́ \lambda \varepsilon \mu o \varsigma-\alpha \sigma \tau \rho \alpha \pi \eta \dot{~: ~} E v \alpha \varsigma \pi o ́ \lambda \varepsilon \mu o \varsigma(\gamma \rho \dot{\eta} \gamma o \rho o \varsigma \sigma \alpha v \tau \eta v) \alpha \sigma \tau \rho \alpha \pi \dot{\eta}$

'guerre-éclair: Une guerre (rapide comme l') éclair'

Examinons enfin la catégorie des locutions verbales afin de dégager les caractéristiques de l'intensité rencontrée dans un certain nombre de locutions de ce type.

\subsection{Les locutions verbales}

Il existe dans les langues des suites du type verbe+compléments où la relation entre le verbe et les compléments n'est pas compositionnelle, qui ont cependant la distri-

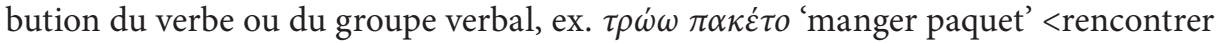
des difficultés énormes>, $\pi \varepsilon \tau \alpha \dot{\alpha} \omega \tau \eta \sigma \kappa o v ́ \varphi \iota \alpha \mu \nu$ 'jeter son bonnet' <avoir une envie

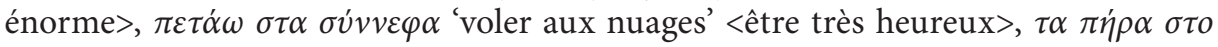

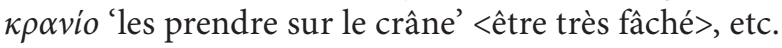

Toutes ces expressions sont des variantes expressives qui coexistent en langue avec d'autres expressions plus neutres signifiant la même chose. Elles ont un caractère affectif et dénotent la position de la personne qui les emploie d'une manière forcée ou marquée. La majorité d'entre elles expriment le haut degré, comme les traductions des exemples cités plus haut le montrent. Comme on l'a déjà noté dans 2 , souvent à côté d'un intensif on trouve un atténuatif qui entre en relation paradigmatique d'antonymie avec l'intensif. Ainsi, on rencontre parmi ce type d'exemples des cas comme:

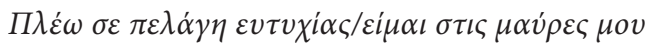

'Naviguer à des mers de bonté/je suis à mes noires'

Ce qui différencie les locutions verbales de tous les autres cas étudiés dans cet article, est le fait qu'elles ne comportent ni terme intensificateur ni terme intensifié. Toute la locution verbale exprime l'idée de l'intensité en étant 'synonyme' ou - mieux - paraphrasée par une expression intensive libre. C'est pour cette raison qu'il est impossible de parler de redondance sémique dans les locutions verbales, dans la mesure où il ne peut pas y avoir une répétition indirecte du terme intensifié à l'aide du terme intensificateur.

Après avoir étudié d'une manière détaillée les caractéristiques de différents types d'expressions intensives, considérons à présent comment il serait possible de résoudre d'une manière efficace les problèmes de traduction mentionnés dans 2.2.

\section{La traduction des expressions intensives}

Nous avons vu plus haut que chaque expression intensive étudiée dans cet article (cf. 3.1, 3.2, 3.3), à l'exception des locutions verbales (cf. 3.3), comprend un terme intensifié et un terme intensificateur. Il est clair que le terme intensificateur constitue un actualisateur du prédicat qui est le terme intensifié.

Buvet (à paraître b) a montré qu'on a besoin de descriptions formalisées des données linguistiques afin de traduire d'une langue à une autre de manière efficace. Pour lui, il faut que les différentes valeurs grammaticales (appelées significations grammaticales) rattachées aux actualisateurs se présentent sous la forme soit d'indice (notamment quand il s'agit d'informations temporelles comme passé, futur, présent...), soit de prédicat métalinguistique (e.g. quand les valeurs sont l'intensité forte ou faible). 
Étudions maintenant comment une représentation métalinguistique du prédicat nous permettrait de traduire les expressions intensives d'une manière efficace. Prenons ici comme exemple le français et le grec. Considérons la phrase suivante:

\section{Il fait un froid de canard}

Les différents constituants de la phrase sont: le nom froid que l'on interprète comme un prédicat; Il qui est un argument zéro; fait et un ..._de canard qui constituent l'actualisation du prédicat. Si nous considérons la signification grammaticale des éléments de cette phrase, nous constatons que le verbe support est non marqué, tandis que le reste de l'actualisation apporte une information supplémentaire d'intensité au prédicat.

Si nous décrivons la phrase ci-dessus d'une manière plus abstraite, nous arrivons à une représentation métalinguistique du type:

RM=INT (froid (il))

(RM: représentation métalinguistique; INT: intensité)

Afin de traduire correctement en grec la phrase donnée plus haut comme exemple, il suffit alors de repérer la représentation métalinguistique équivalente pour le prédicat $\kappa \rho v i o$ 'froid', ce qui va générer la phrase:

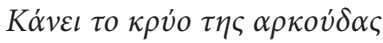

$<$ Il fait le froid de l'ours $>$

Prenons maintenant un exemple du grec. Considérons la phrase suivante:

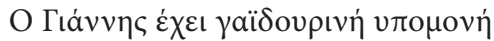

$<$ Jean a une patience d'âne>

Dans cette phrase, le prédicat $v \pi \circ \mu \nu v \eta \dot{~ ' p a t i e n c e ' ~ e s t ~ a c t u a l i s e ́ ~ p a r ~ l e ~ v e r b e ~ s u p p o r t ~}$ $\varepsilon \chi \chi \omega$ 'avoir' et sélectionne $\Gamma \iota \dot{\alpha} v v \eta \varsigma$ 'Jean' en tant qu’argument zéro. Le modifieur adjec-

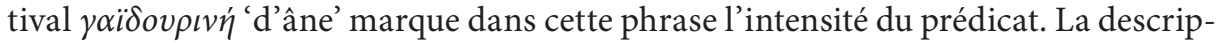
tion métalinguistique de cette phrase serait la suivante:

RM=INT (

La représentation métalinguistique équivalente en français, va générer la phrase:

Jean a une patience d'ange

Comme Buvet (à paraître b) l'a montré, cette manière de procéder dans la traduction d'une langue à une autre "présente l'avantage de proposer des équivalents de traduction qui ne sont pas dépendants des formes de départ de la langue source et de rattacher toutes sortes de constructions donnant lieu à des phrases synonymes à une même représentation métalinguistique». De plus, il s'agit d'un procédé économique, dans la mesure où on n'est pas obligés de prévoir des entrées séparées dans les dictionnaires électroniques de systèmes de traduction automatique pour chaque expression comprenant un modifieur figé; il suffit simplement de marquer les informations concernant l'actualisation dans l'entrée pour chaque prédicat.

Mentionnons, toutefois, que la description métalinguistique ne peut pas être appliquée dans la traduction de locutions verbales intensives (étudiées en 3.3) dans la mesure où, dans ces exemples, il est impossible de repérer le prédicat et l'actualisateur. 


\section{Conclusion}

Dans cet article, nous avons étudié trois cas d'expressions figées intensives: les déterminants figés, les suites du type NN et les locutions verbales. Nous avons constaté que:

a) d'un point de vue sémantico-syntaxique, certaines suites $\mathrm{NN}$ se comportent en tant que déterminants figés;

b) l'expression de l'intensité dans les locutions verbales est tout à fait différente de celle rencontrée dans les déterminants figés et les suites $\mathrm{NN}$, dans la mesure où dans le premier cas il est impossible de trouver le terme intensificateur et le terme intensifiant;

c) il serait possible d'arriver à une traduction efficace des expressions intensives d'une langue à l'autre, si on marque les différentes valeurs rattachées aux actualisateurs sous forme de représentation métalinguistique.

Pour avoir une vue complète sur le phénomène d'intensité dans les expressions figées et les problèmes éventuels de traduction liés à l'intensité, il reste encore à : a) étudier tous les types d'expressions figées afin de dresser la liste des structures susceptibles d'exprimer l'intensité et de décrire d'une manière détaillée la façon dont elles le font; b) proposer une solution pour la traduction des locutions verbales intensives dans plusieurs langues.

\section{NOTES}

1. Pour les termes intensif, intensité, cf. par ex. le dictionnaire de Mounin (1974): «intensif: On appelle intensif une forme linguistique (souvent dérivée), qui donne au sens d'une forme voisine une intensité plus forte, qui la porte à un degré plus élevé: les adverbes comme très et énormément sont des intensifs» ou le dictionnaire de Dubois et al. (1994): «intensif: On appelle intensifs les noms, adjectifs, adverbes ou verbes formés avec un préfixe ou un suffixe qui indique un degré élevé de la propriété indiquée par la racine. Les préfixes ultra, extra, super, sur, etc., ont servi à former des intensifs. »

2. Pour elle, un intensificateur est un élément comme très.

3. Selon le modèle théorique Sens-Texte, le concept de fonction lexicale permet de rendre compte de deux types de relations lexicales distinctes: les dérivations sémantiques (qui sont les fonctions lexicales paradigmatiques comme Syn pour la synonymie, etc.) et les relations base-collocatif (qui sont les fonctions lexicales syntagmatiques comme Magn pour l'intensification, Oper pour les verbes supports, etc.).

4. La phrase subjonctive est ici l'équivalente de la construction infinitive du français.

5. En grec moderne, chaque préposition impose un cas particulier au nom qu'elle gouverne. La préposition $\alpha \pi o ́$ ' $d e$ ' impose l'accusatif au nom qui la suit.

6. Toutefois, nous avons recensé un cas où cette constatation n'est pas valable: quand le déterminant nominal figé $\omega \kappa \varepsilon \alpha v o ́ \varsigma$ 'océan', qui désigne une grande quantité se combine avec le substantif $\iota \delta \rho \omega ́ \tau \alpha \varsigma$ 'sueur' en position N2, ce N2 reste au singulier. En français, il existe également des contreexemples: un Himalaya de bêtise.

7. Le grec moderne préfère la structure NN (affiche-géant) à NA (affiche géante).

\section{RÉFÉRENCES}

Benninger, C. (2001): «Une meute de loups / une brassée de questions: collection, quantification et métaphore», Langages 129, pp. 21-34.

Berthelon, C. (1955): L'expression du haut degré en français contemporain, Francke, Berne.

Blanco, X. (1997): «Un dictionnaire électronique des déterminants nominaux en espagnol», Actes du Colloque International FRACTAL 1997, BULAG, pp. 45-53.

Blanco, X. (1996): «Los complementos de nombre intensivos. Enfoque contrastivo españolfrancés », Revista de Filología Francesa 10, Publicaciones Universidad Complutense, Madrid, pp. 55-67. 
Blanco, X. (2002): «Les déterminants figés», Langages 145, pp. 61-80.

Blanco, X., Buvet, P-A. et Z. Gavrillidou (1999): «Analyse comparée des modifieurs figés en espagnol, français, grec. Vers un dictionnaire électronique», in Studies in Greek linguistics, Proceedings of the 20th Annual Meeting of the Department of Linguistics, Aristotle University of Thessaloniki.

Buvet, P-A. (1993): Les déterminants nominaux quantifieurs, thèse de doctorat, Paris, Université Paris 13.

Buvet, P-A. (1995) : «Les compléments de nom intensifs», Néophilologica 12, Katowice, Université de Silésie.

Buvet, P-A. (à paraître a): Remarques sur la détermination du français.

Buvet, P-A. (à paraître b): «Détermination et figement», Meta.

Buvet, P-A. et G. Gross (1995), «Comparaison et expression du haut degré dans le groupe nominal», Faits de Langue 5, Paris, PUF.

Cruse, D.A. (1986): Lexical Semantics, Cambridge University Press.

De Cesare, A-M. (2002): Intensification, modalisation et focalisation. Les différents effets des adverbes proprio, davvero et veremente, Peter Lang.

Flaux, N. (1999): «A propos des noms collectifs», Revue de linguistique romane 63, pp. 471-502.

GaVrillidou, Z. (1997): Étude comparée des suites NN en français et en grec. Élaboration d'un lexique bilingue, Lille, Presses Universitaires du Septentrion.

Gavrillidou, Z. (1998a): «Les déterminants nominaux figés en grec moderne. Une première approche», Actes des Premières Rencontres Linguistiques Méditerranéennes sur le figement lexical, Tunis, pp. 35-50.

Gavrillidou, Z. (1998b): «Un cas de figement: les compléments du nom intensifs en grec moderne. Étude comparée grec-français-espagnol», Besançon, BULAG 23, pp. 89-110.

Gavrillidou, Z. (2002): «Structures Dét N1 N2 et Détermination Figée», in Blanco, X., Buvet, P.-A. et Z. Gavrinlidou (dir.), Détermination et Formalisation, Linguisticae Investigationnes Supplementa 23, Amsterdam/Philadelphia, John Benjamins.

Gavrillidou, Z. (2004): "Verbes supports et intensité», Linguisticae Investigationnes 27-2, Amsterdam/Philadelphia, John Benjamins.

Gross, G. (1988): «Degré de figement des noms composés», Langages 90, Paris, Larousse, pp. 57-72.

Gross, G. (1992): «Forme d'un dictionnaire électronique», L'environnement traductionnel, Sillery-Montréal, Presses de l'Université du Québec, Aupelf-Uref.

Gross, G. (1996): Le figement en français. Noms composls et autres locutions, Ophrys, Paris.

Hunston, S. and G. Thompson (2000): Evaluation in Text, Oxford, Oxford University Press.

Lаккоғ, G. (1987): Women, Fire and Dangerous Things, Chicago, University of Chicago Press.

Leroy, S. (2004): «Sale comme un peigne et méchant comme une teigne. Quelques remarques sur les comparaisons à parangon», Travaux Linguistiques du Cerlico 17, pp. 255-267.

MejRI, S. (1994): «Séquences figées et expression de l'intensité. Essai de description sémantique», Cahiers de lexicologie 65, pp. 111-122.

MejRI, S. (1996): Le figement lexical, Thèse d'Etat, Université des Lettres, La Mannouba.

MeL'Čuk, I., (1988): "Collocations and lexical functions», in Cowie, A.-P. (ed), Phraseology. Theory, Analysis and applications, Oxford, Clarendon Press.

MeL'Čuk, I., (1997): Vers une linguistique Sens-Texte, Leçon inaugurale, Collège de France.

Polguère, A. (1998) : «Pour un modèle stratifie de la lexicalisation en génération de texte», TAL 39-2.

Romero, C. (2001): L'intensité en français contemporain. Analyse sémantique et pragmatique, thèse de doctorat, Université Paris 8.

SCAPIRA, Ch. (2000): «Du prototype au stéréotype, et inversement: le cliché 'comme+SN'», Cahiers de lexicologie 76, pp. 27-40.

Szende, T. (1999), «A propos des séquences intensives stéréotypés: Plaidoyer pour une description lexicographique», Cahiers de lexicologie 74, pp. 61-77.

ТАмва, I. (1981): Le sens figuré, Paris, PUF. 